\title{
Relative Brain Size and Cognitive Equivalence in Fishes
}

\author{
Zegni Triki ${ }^{a} b$ Mélisande Aellen ${ }^{a}$ Carel P. van Schaik ${ }^{c}$ Redouan Bshary ${ }^{a}$ \\ aBehavioural Ecology Laboratory, Faculty of Science, University of Neuchâtel, Neuchâtel, Switzerland; ${ }^{\mathrm{b}}$ Institute of

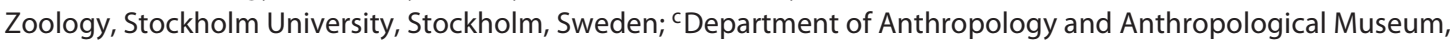 \\ University of Zurich, Zurich, Switzerland
}

\section{Keywords}

Encephalization quotient - Cognitive equivalence . Intelligence $\cdot$ Brain size $\cdot$ Body size $\cdot$ Slopes $\cdot$ Cognitive performance $\cdot$ Cleaner fish

\begin{abstract}
Scientists have long struggled to establish how larger brains translate into higher cognitive performance across species. While absolute brain size often yields high predictive power of performance, its positive correlation with body size warrants some level of correction. It is expected that larger brains are needed to control larger bodies without any changes in cognitive performance. Potentially, the mean value of intraspecific brain-body slopes provides the best available estimate for an interspecific correction factor. For example, in primates, including humans, an increase in body size translates into an increase in brain size without changes in cognitive performance. Here, we provide the first evaluation of this hypothesis for another clade, teleost fishes. First, we obtained a mean intraspecific brain-body regression slope of 0.46 (albeit with a relatively large range of $0.26-0.79$ ) from a dataset of 51 species, with at least 10 wild adult specimens per species. This mean intraspecific slope value (0.46) is similar to that of the encephalisation quotient reported for
\end{abstract}

karger@karger.com www.karger.com/bbe

Karger"
(C) 2021 The Author(s)

Published by S. Karger AG, Basel

This is an Open Access article licensed under the Creative Common Attribution-NonCommercial-4.0 International License (CC BY-NC) (http://www.karger.com/Services/OpenAccessLicense), applicable to the online version of the article only. Usage and distribution for commercial purposes requires written permission. teleosts (0.5), which can be used to predict mean cognitive performance in fishes. Importantly, such a mean value (0.46) is much higher than in endothermic vertebrate species $(\leq 0.3)$. Second, we used wild-caught adult cleaner fish Labroides dimidiatus as a case study to test whether variation in individual cognitive performance can be explained by body size. We first obtained the brain-body regression slope for this species from two different datasets, which gave slope values of 0.58 (MRI scan data) and 0.47 (dissection data). Then, we used another dataset involving 69 adult cleaners different from those tested for their brain-body slope. We found that cognitive performance from four different tasks that estimated their learning, numerical, and inhibitory control abilities was not significantly associated with body size. These results suggest that the intraspecific brain-body slope captures cognitive equivalence for this species. That is, individuals that are on the brain-body regression line are cognitively equal. While rather preliminary, our results suggest that fish and mammalian brain organisations are fundamentally different, resulting in different intra- and interspecific slopes of cognitive equivalence.

2021 The Author(s)

Published by S. Karger AG, Base 


\section{Introduction}

Scholars have long sought regularities in the relationship between brain size and body size. These analyses, largely focussed on mammals, showed an unexpected pattern: the slope of (log-transformed) brain size on (logtransformed) body size is often lower within than between species and higher taxa [Pilbeam and Gould, 1974; Pagel and Harvey, 1988; Tsuboi et al., 2018]. Two different explanations for this phenomenon, called the taxonlevel effect, have been proposed. The first attributes the higher slope at higher taxonomic levels to the combined effect of two parallel macroevolutionary processes: Cope's rule [Alroy, 1998], the tendency for more recently evolved lineages to have larger bodies than their forebears, and Lartet-Marsh's rule [Jerison, 1973; Halley and Deacon, 2017], the tendency for more recently evolved lineages to have larger brains at a given body size than their forebears. The combined effect of the two processes would be to artificially inflate the regression slope through the total sample of all species relative to those within a given species.

A second explanation is based on statistical logic: the estimated slope is reduced when the error in body size measures is greater than that for brain size [see detailed discussion in van Schaik et al., 2021]. This effect is strongly affected by the body size range in the sample and thus far greater within species than between them. This phenomenon has been argued to explain why estimates for within-species slopes show extensive variation [Pilbeam and Gould, 1974], even among different mammalian lineages [Martin and Harvey, 1985].

While the two explications for the taxon effect should in principle apply to all taxa, Tsuboi et al. [2018] showed that there is a clear pattern in this variation: ectothermic vertebrates (fishes, amphibians and reptiles) have far steeper intraspecific slopes (in their sample approximately 0.50$)$ than the endothermic birds and mammals (0.150.20 ). Because the majority of ectotherms show indeterminate growth and, thus, a far greater range of adult body sizes, the error problem might, in principle, explain this difference. However, Tsuboi et al. [2018] showed that the error problem was unlikely to explain this difference fully. Indeed, a recent analysis that used sexually dimorphic primates to calculate intraspecific slopes using mean values for males and females, and so minimize the error problem, obtained only moderately steeper slopes of 0.25-0.3 [van Schaik et al., 2021]. Nevertheless, it remains unclear what value we should expect for the ectotherms for 2 reasons. First, the full dataset used by Tsuboi et al.
[2018] contained both wild and captive fishes, but hatchery-reared fish have smaller brains compared to fish of the same species in the wild [see review by Huntingford, 2004]. Second, they did not analyse how much the range of body sizes in a species' sample affected the value of the slope. This is important because fishes have a much larger range of body sizes than birds or mammals due to their indeterminate growth [Froese and Froese, 2019]. This feature makes fishes more suitable than birds or mammals to address these statistical issues. Therefore, the first aim of this paper is to refine the findings of Tsuboi et al. [2018] by estimating intraspecific slopes using only wildcaught individuals and assessing the effect of intraspecific variation in adult body size on the estimate. To do so, we identified 51 fish species for which sample sizes of wild specimens were large enough ( $\geq 10$ data points) to calculate brain-body slopes.

Suppose the analyses corroborate the difference between endothermic and ectothermic vertebrates in the slope of the brain-body relationship. In that case, this raises the important question of how the different slopes translate into cognitive performance. We follow Shetlleworth [2010], who defined cognition as the ability to acquire, process and retain information and act on it. This broad definition of cognition may justify analyses on total brain size, while specific areas should be studied when their function is known, like the hippocampus and spatial memory [Krebs et al., 1989]. Regarding total brain size analyses, Jerison [1973] had proposed the encephalization quotient (called EQ), the ratio of a species' actual brain size to its predicted brain size based on its cladespecific brain-body regression line, to capture its cognitive performance relative to other species within the same clade. The use of EQ as a predictor for relative cognitive performance across species has been criticised largely because of the taxon-level effects reported for endothermic vertebrates [van Schaik et al., 2021]. Indeed, EQ is a poor predictor of cognitive performance in mammals, with absolute brain size being a better predictor [Rensch, 1973; Deaner et al., 2007]. However, the use of absolute brain size ignores evidence that larger bodies warrant larger brains for body maintenance [Martin and Harvey, 1985]. Therefore, intraspecific slopes have been proposed as an alternative predictor of cognitive performance across mammals [van Schaik et al., 2021]. The key idea of this alternative approach is that the intraspecific slope reflects the extra amount of brain tissue required to sustain the additional somatic functions in larger individuals because there are no changes in bauplan and sensory-motor abilities. The critical assumption thus is that the cognitive 
performance of adult individuals within a species is independent of their body size. This assumption can be argued to hold for humans: one good estimate of overall cognitive performance, IQ, shows only a very modest effect of the sex difference in body size [Irwing and Lynn, 2005]. In addition, cognitive test batteries applied to various species find no effects of body size, including sex differences, among dimorphic species [reviewed in van Schaik et al., 2021; see also Bohn et al., 2021]. Therefore, the intraspecific brain-body slope can potentially also serve to identify the line of so-called "cognitive equivalence" across species [van Schaik et al., 2021], at least within more recent lineages that share a similar brain bauplan, like neuron densities [Herculano-Houzel, 2017]. The prediction will be that if two species, for example, have similar intraspecific brain-body slopes and intercepts, they would eventually be cognitively equal. If, in another scenario, the two species still have similar slopes but different intercepts, the species with the higher intercept should cognitively outperform the one with a lower intercept [van Schaik et al. 2021]. Nevertheless, this prediction remains to be tested. Furthermore, the markedly higher value of the intraspecific slope in ectotherms than endotherms raises the question of whether adults of the same ectotherm species but with different body sizes also show cognitive equivalence or whether the larger and hence older individuals outperform smaller ones.

Therefore, the second aim of this study is to provide a first test of the prediction that the cognitive performance of adult individuals within a fish species is not correlated with body size (which is also a proxy of age in ectotherms). Although we know little about domain-general intelligence in ectotherms [Poirier et al., 2020; Aellen et al., 2021, preprint], unlike in endotherms [Burkart et al., 2017], we can examine whether a variety of cognitive tests show a body size effect on performance. If they all yield no correlation with body size, we can assume cognitive equivalence in our study species, amenable to further testing in other fish species.

We examined the relationship between cognitive performance and body size in adult cleaner fish Labroides dimidiatus, where we also provide the slope of the brainbody regression. Cleaner fish engage in iterative mutualistic interaction with a variety of coral reef fish species (hereafter "clients"). Cleaners remove clients' ectoparasites and dead tissue in exchange for food [Losey, 1979], a behaviour that is mutually beneficial for both cleaner [Grutter, 1999] and client [Clague et al., 2011; Waldie et al., 2011; Demairé et al., 2020; Ros et al., 2020]. Cleaner fish show a highly sophisticated strategic behaviour that includes reputation management [Bshary and Grutter, 2002; Binning et al., 2017], social tool use [Bshary et al., 2002], reconciliation [Bshary and Würth, 2001], and social competence [Triki et al., 2020a]. Cleaner fish may outperform primates in some learning tasks [Salwiczek et al., 2012] that go beyond conditioning [Quiñones et al., 2020]. They also show evidence for generalised rule learning [Wismer et al., 2016], numerical discrimination [Triki and Bshary, 2018], long-term memory [Triki and Bshary, 2019], and mirror self-recognition [Kohda et al., 2018].

We used published data on cleaner fish cognitive performance in 4 different cognitive tasks [Aellen et al., 2021, preprint] to explore the relationship between body size and cognitive performance. These results were then combined with estimates of the brain-body slope in this species using published data from two studies on brain size [Triki et al., 2019a; 2020a]. These datasets also contain information regarding the size of five major brain areas, that is, telencephalon, diencephalon, midbrain, cerebellum and brainstem. As these areas differ in their relative importance to subparts of cognitive performance (e.g., perception vs. decision-making vs. execution of actions [Striedter, 2005]), we also explored the regression slopes of the five main brain region sizes on body size in cleaner fish. The brain data and the cognitive performance data were not collected from the same individuals. Thus, these datasets are independent, although a dependent dataset would have been preferable to test whether residuals from the brain-body regression would predict cognitive performance. Nevertheless, the data we have in this study show to what extent intraspecific brain-body slopes in fishes may reflect cognitive equivalence.

\section{Materials and Methods}

Brain and Body Size Data from Other Fish Species

We compiled fish brain and body size data from the database FishBase [Froese and Pauly, 2019] and the published study by Tsuboi et al. [2018]. We selected only confirmed wild-caught individuals and discarded every dataset from lab-reared fish or fish obtained through aquaria trade. Furthermore, we selected only adult individuals based on the adult body size for the species [Tsuboi et al., 2018; Froese and Pauly, 2019]. Finally, species were included when at least 10 individual measurements of body and brain size were reported. Using these rigorous selection criteria, we could include 52 species, with sample sizes ranging from 10 to 60 individuals (the exact sample size per species is reported in Table 1).

To estimate every species' log brain-log body regression slope from the fishes' dataset, we fitted a set of linear models (LMs, with ordinary least squares [OLS] approach). We validated these LMs 
Table 1. Brain-body slope estimate of the 51 fish species with $95 \% \mathrm{Cl}$

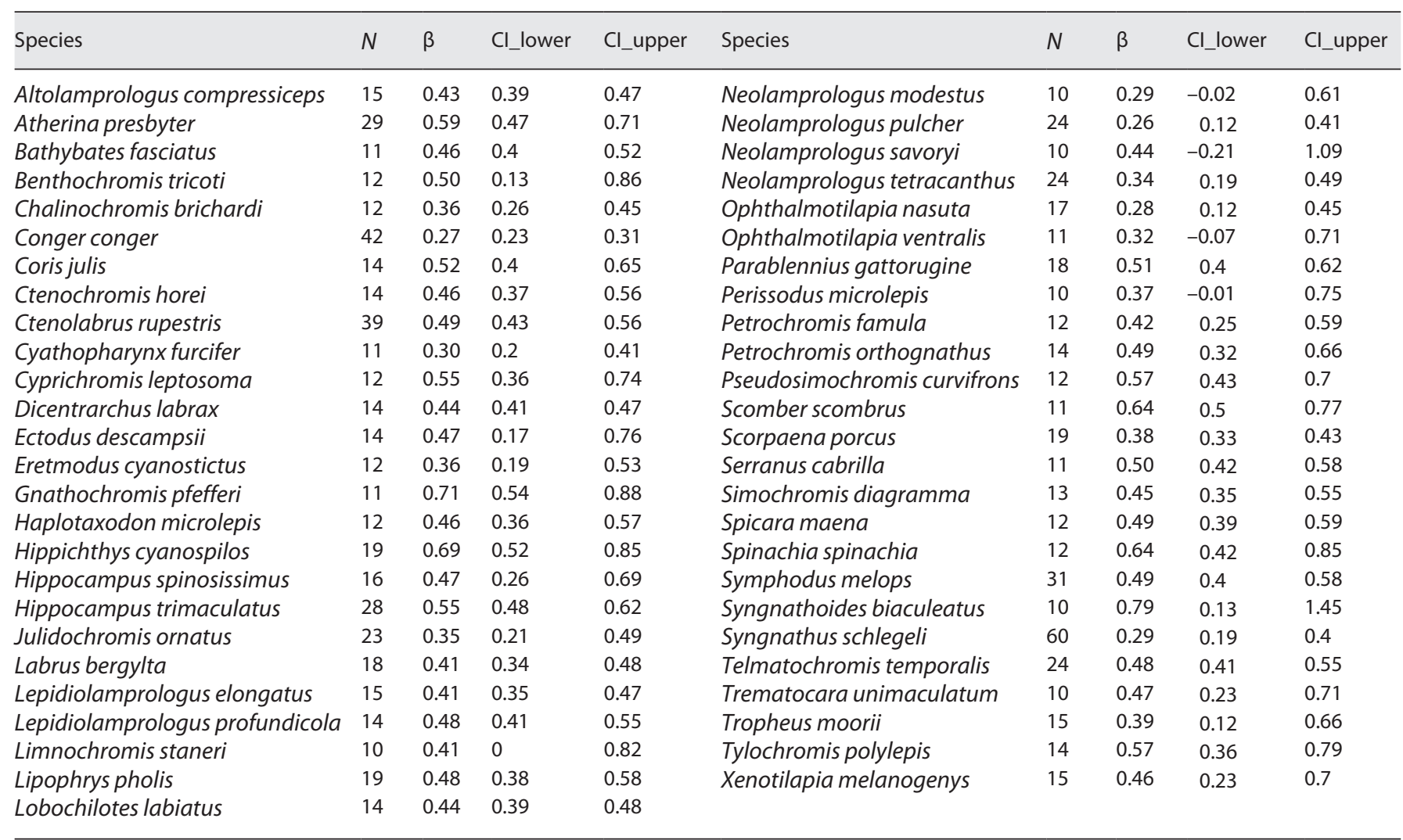

by checking model assumptions like the normal distribution and homogeneity of variance of the residuals. Furthermore, we estimated the range of body sizes in a given species' dataset for each of the 52 (below it gets to be 51) species by simply calculating the body size (as body mass in g) ratio of the largest (i.e., heaviest) to the smallest (i.e., lightest) individual. We then tested whether there is an effect of body size ratio on these species' brain-body slopes. To do so, we fitted a phylogenetic generalised least-squares regression (PGLS) of log brain-log body slope (i.e., response variable) on logtransformed body size ratio (i.e., continuous predictor). We also explored whether sample size (log-transformed number of individuals per species) affected the estimated brain-body slopes by fitting a PGLS. The PGLS approach considers the phylogenetic relationships to produce an estimate of expected covariance in crossspecies data. For this, we estimated Pagel's $\lambda$ (lambda) as the phylogenetic signal in PGLS models. Pagel's $\lambda$ is an estimate of how correlations in given traits reflect their shared evolutionary history cross-species. To calculate Pagel's $\lambda$, we ran a maximum likelihood function for each PGLS model separately (see code for further details). For the PGLS models to meet the assumptions of normal distribution and homogeneity of variance, we had to exclude one species (Neolamprologus multifasciatus) from the analyses because it had studentized residuals larger than 3 [Jones and Purvis, 1997], thus reducing our sample size to 51 fish species.
We ran a phylogenetic signal analysis to test whether Pagel's $\lambda$ of the brain-body slope is significantly different from zero (the null hypothesis). A significant $\lambda>0(p \leq 0.05)$ would mean that phylogeny affects the value of the estimated brain-body slopes. Furthermore, we ran a multiple comparisons analysis on the 51 fish slopes by fitting a global model for brain-body size with species as a factor. Post hoc tests with the Tukey correction method helped in comparing brain-body slopes between all these fish species. A detailed step-by-step code is provided as a script file that runs in the open-source statistical software R [R Core Team, 2020] (see Data Availability Statement).

\section{Case Study: Brain Size, Body Size and Cognitive Performance}

Data in L. dimidiatus

We used published data on $L$. dimidiatus as a case study to test the link between brain size and body size, on the one hand, and cognitive performance and body size, on the other hand. There were no data on brain size for the animals whose cognitive performance was estimated. All the data we use here is from fish collected on the reef around Lizard Island, Great Barrier Reef, Australia, between 2016 and 2019. Cleaner fish have a lifespan of about 5 years [Eckert, 1987]. They undergo a pelagic larval phase before settling on a coral reef [Victor, 1986]. Therefore, all fish sampled in these datasets belong to the same population [see Triki et al., 2018]. 
Brain Size Data

We compiled datasets from published studies by Triki et al. [2019a; 2020b]. These 2 studies were conducted on wild-caught female cleaner fish $L$. dimidiatus at Lizard Island. Both studies collected fish body size as body weight to the nearest $0.01 \mathrm{~g}$ but differed with respect to the method used to quantify brain size: Triki et al. [2019a] used the magnetic resonance image (MRI) technique to estimate brain volume (in $\mathrm{mm}^{3}$ ) of $n=15$ female cleaner fish, while Triki et al. [2020a] manually dissected and weighed the brains of $n=18$ female cleaner fish to the nearest $0.1 \mathrm{mg}$ (for further details, please refer to the original studies by Triki et al. [2019a; 2020a]). These datasets also had the volume/mass of the 5 major regions of fish brains, that is, telencephalon, diencephalon, midbrain, cerebellum and brain stem.

\section{Cognitive Performance Data}

We compiled data from the study by Aellen et al. [2021, preprint]. The dataset comprises cognitive performance of $n=69$ wild-caught female cleaner fish tested in 4 different laboratory tasks at Lizard Island, Great Barrier Reef, in Australia. Here, we used these data to explore the relationship between body size and cognitive performance. Below, we provide brief descriptions for each cognitive task, but for more details, please refer to the original study by Aellen et al. [2021, preprint]. Note that given the nature of cleaner fish feeding behaviour (i.e., removing ectoparasites off the skin of client fish), researchers often feed them in captivity with a paste of mashed prawn or a mixture of fish flakes and mashed prawn smeared as a thin layer on Plexiglas plates. Such plates with smeared food paste can be viewed as surrogates for client fish skin with ectoparasites in laboratory settings.

Reversal Learning Task. Aellen et al. [2021, preprint] tested fish learning and flexibility abilities in a reversal learning task. The experiment was based on a simultaneous 2-choice task with Plexiglas plates with colour and shape cues, that is, yellow triangular versus green round plates. Fish first had to learn the association between the yellow triangle and a food reward. As soon as fish had learned this association, the reward contingency was reversed, and the previously non-rewarding green plate became the one with the food reward. At this stage, fish were offered a maximum of 100 trials (i.e., 10 sessions of 10 trials each over 5 consecutive days) to learn the reversal association. The learning criterion set for this task is that each individual has to significantly $(p \leq 0.05)$ learn the rewarding colour above chance level (50\%). That is, successful fish were those that scored either 7 correct choices out of 10 in 3 consecutive test sessions (i.e., 21/30), 8 correct choices out of 10 in 2 consecutive sessions (i.e., 16/20), or at least 9 correct choices out of 10 in a single session (i.e., 9/10).

Detour Task. The detour task aimed to estimate fish inhibitory control abilities. Here, a novel Plexiglas plate with a food reward was placed behind a transparent barrier. To access the food reward, fish had to inhibit their impulses to go straight to the visible food and instead swim around the barrier to reach the food. Bumping into the barrier, however, shows that the subject is impatient to get the food. Therefore, this measure (i.e., number of head bumps) provides an estimate of inhibitory control abilities, where fewer head bumps indicate better abilities. In total, Aellen et al. [2021, preprint] tested fish inhibitory control abilities in 10 detour trials. The estimated performance was the average number of fish head bumps on the barrier until reaching the food.
Numerical Discrimination Task. Aellen et al. [2021, preprint] used Plexiglas plates with various numerical quantities as black squares against a white background to test for fish numerical abilities. The number of black squares ranged from 1 to 9. The task consisted of presenting two Plexiglas plates at a time with two different quantities of black squares. In total, there were 20 different combinations of the 9 quantities. The general rule was that, in every combination, the plate depicting the largest quantity of black squares was always the rewarding plate with food items. The performance consisted of the proportion of correct choices from 160 test trials over eight days.

Feeding against Preferences Task. During the cleaner-client cleaning interaction, cleaner fish often prefer to bite client mucus instead of cooperating and eating ectoparasites [Grutter and Bshary, 2003]. Such events can be reproduced in laboratory conditions with Plexiglas plates as surrogates for clients, and fish flakes and mashed prawn as surrogates for client ectoparasite and mucus, respectively [Bshary and Grutter, 2006]. Aellen et al. [2021, preprint] used a similar laboratory set-up to test whether cleaner fish can inhibit their food preferences for prawn and feed on fish flakes instead. A Plexiglas plate with two fish flakes items and two prawn items was presented to the fish during test trials. The plate remained in the aquarium as long as fish fed on flakes items (i.e., a less preferred food as an indicator of inhibition of preferences). However, the plate was withdrawn from the aquarium upon eating a prawn item (i.e., a highly preferred food as an indicator of noninhibition of preferences). Thus, the optimal feeding strategy to maximise food intake is to feed first on the less preferred food (flakes) before eating one highly preferred food item (prawn). We then estimated fish performance in this task as a ratio reflecting the degree of feeding against preference. To do so, we divided the total number of fish flakes items by the total of prawn items consumed throughout the 12 test trials. Next, we fitted a linear regression model of feeding ratio on log-transformed body weight for the cognition-body slope from this data.

\section{Data Analyses for the Cleaner Fish Brain Data}

We used the cleaner fish brain data to calculate the slope of brain size (either as volume or mass) as a function of body size and the regression slope of every brain part on body size. To do so, we fitted LMs (with OLS) in the open-source statistical software R [R Core Team, 2020] of log-transformed brain measurement on logtransformed body mass. We had two separate sets of LMs, given that brain morphology was estimated once as a volume and once as a mass via MRI and manual dissection methods, respectively. We validated all these LMs by checking model assumptions like the normal distribution and homogeneity of variance of the residuals.

We ran a generalised linear model (GLM) with quasipoisson distribution error for data analyses linked to the cognitive tasks to estimate the regression slope of cognitive performance on logtransformed body weight (g). We ranked individual performance with respect to the performance of all tested fish. In all tasks, we ranked fish in a way that higher rank values correspond to better performance. We used rank data as cognitive performance (i.e., response variable) in the fitted GLMs, where log-body size was the continuous predictor. We fitted GLMs with quasipoisson to solve an overdispersion issue with the Poisson distribution error. 


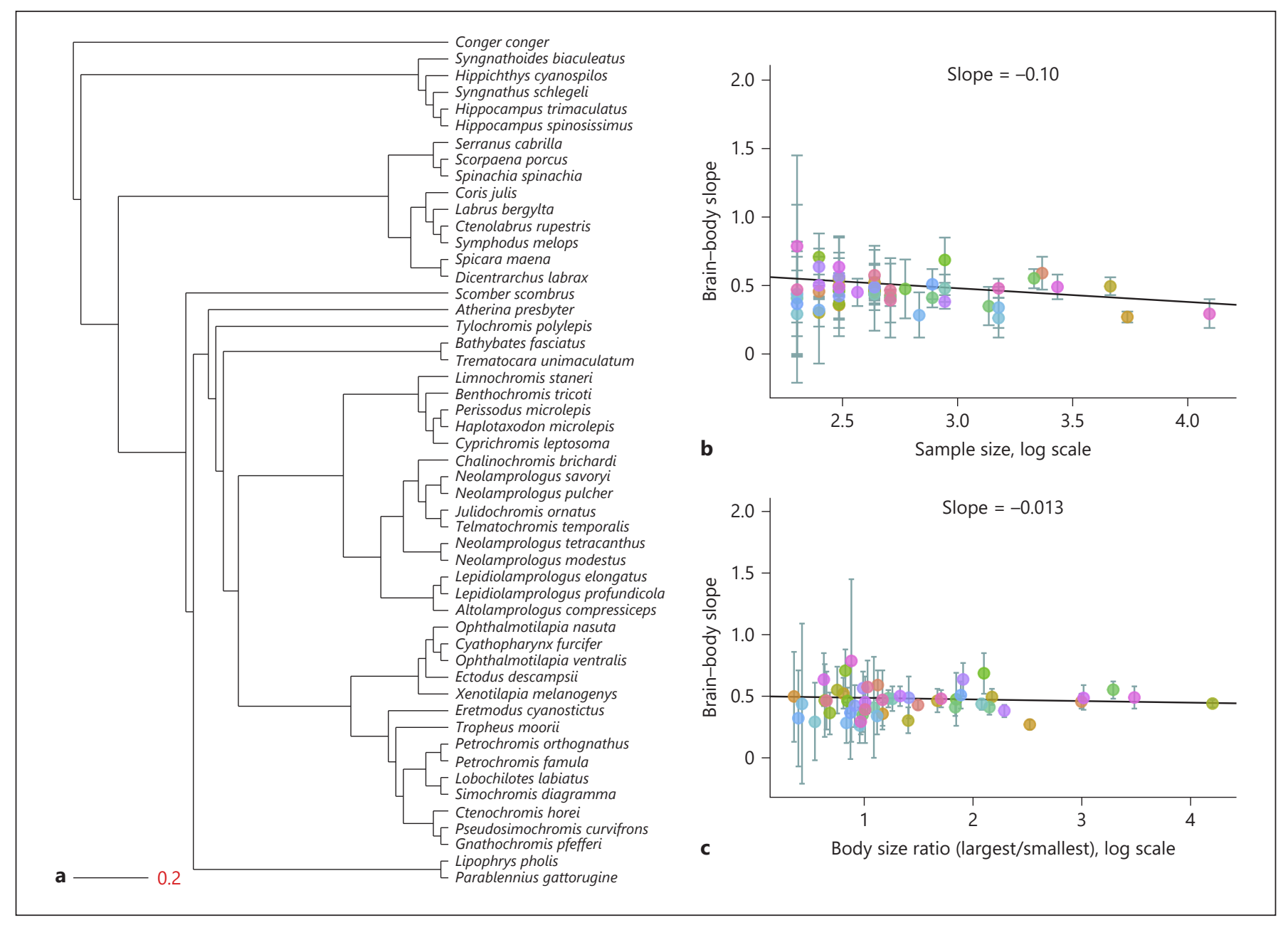

Fig. 1. a Phylogenetic tree of the 51 fish species with a scale indicating tree distance. Relationship of brain-body slope and either sample size used to estimate the brain-body slope (b) or body size ratio (c). The plots show the brain-body slope estimate with 95\% CI as error bars for each species. Brain and body data were compiled from the fish database FishBase [Froese and Pauly, 2019] and from Tsuboi et al. [2018].

\section{Results}

The Intraspecific Brain-Body Slope in Fishes

We calculated intraspecific slopes in wild-caught individuals from 51 fish species (Fig. 1a). These slopes ranged from 0.26 to 0.79 (Table 1), with a mean value of 0.46 and a median also of 0.46 . Sample size (the number of individual data points per species) did negatively affect the brain-body slope estimates, though the effect size was relatively small (PGLS: $n=51, \beta=-0.105, t$ value $=-2.846$, $p=0.006,95 \%$ CI $[-0.18$ to -0.03$], R^{2}=0.13, \lambda=0.55$; Fig. 1b). Most importantly, we found no effect of body size ratio on the brain-body slope estimates (PGLS: $n=$ $51, \beta=-0.013, t$ value $=-0.682, p=0.498,95 \%$ CI $[-0.05$ to 0.03$], \lambda=0.47$; Fig. 1c). Further analyses showed that there was no significant phylogenetic signal in the brainbody slopes ( $p$ value of the likelihood ratio test $=0.16$ ) Nevertheless, the multiple comparisons analysis of fish slopes indicated that some fish species had significantly different brain-body slopes from each other (Fig. 2).

\section{Cleaner Fish Slopes}

Our two datasets used to calculate the slope for the relationship between log brain size and log body size yielded values of 0.58 (MRI scans, LM: $n=15, \beta=0.585, t$ value $=6.133, p<0.001,95 \%$ CI $[0.38-0.79]$; Fig. 3a) and 0.47 (dissection data, LM: $n=18, \beta=0.473, t$ value $=$ $3.593, p=0.002,95 \%$ CI [0.19-0.75]; Fig. 4a), respective- 


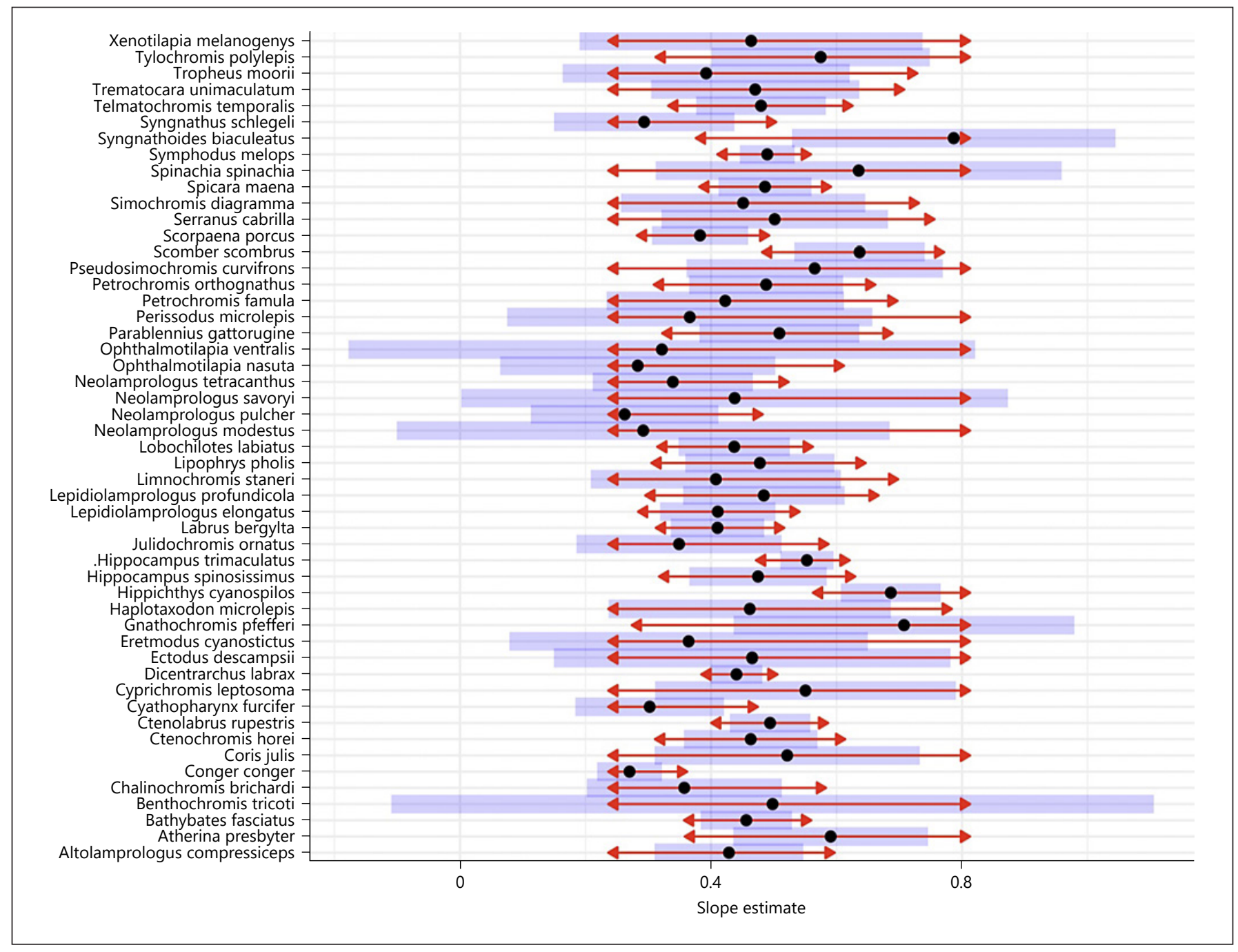

Fig. 2. Comparison of the 51 fish slopes. Black dots are the slope estimate of log-transformed brain size on logtransformed body size. Blue bars are the $95 \%$ CI estimated from the multiple comparisons. The red arrows are indicators for the statical comparisons. If an arrow from one slope estimate overlaps with an arrow from another species, the difference is not statistically significant. We used Tukey methods to adjust for multiple comparisons with an alpha set at 0.05 .

ly. Furthermore, the regression slopes of every major brain part (i.e., telencephalon, diencephalon, midbrain, cerebellum and brain stem) on body size are reported in Table 2 and Figures 3 and 4 . The goodness of fit seems to be sensitive to the method used to estimate the size of these regions. The MRI methods yielded the best estimates with a narrower 95\% CI compared to the values from the manual dissection data (Fig. 3, 4). Overall, brain part slopes showed some variation but were not fundamentally different from the total brain slopes. For example, the telencephalon to body size slopes of 0.54 with
MRI and 0.49 with manual dissection are similar to the total brain's slopes (Fig. 3b, 4b).

For the cognition-body slopes, there was no significant relationship between body size and performance in any of the 4 cognitive tasks, nor did the 4 slopes yield the same (positive or negative) sign: reversal learning (GLM: $n=$ $69, \beta=-0.400, t$ value $=-1.456, p=0.15,95 \%$ CI $[-0.94$ to 0.14]; Fig. 5a), detour task (GLM: $n=69, \beta=0.180, t$ value $=0.642, p=0.523,95 \%$ CI [ -0.37 to 0.73$]$; Fig. $5 b)$, numerical discrimination task (GLM: $n=69, \beta=0.208, t$ value $=0.745, p=0.459,95 \%$ CI $[-0.34$ to 0.76$]$; Fig. 5 c), 


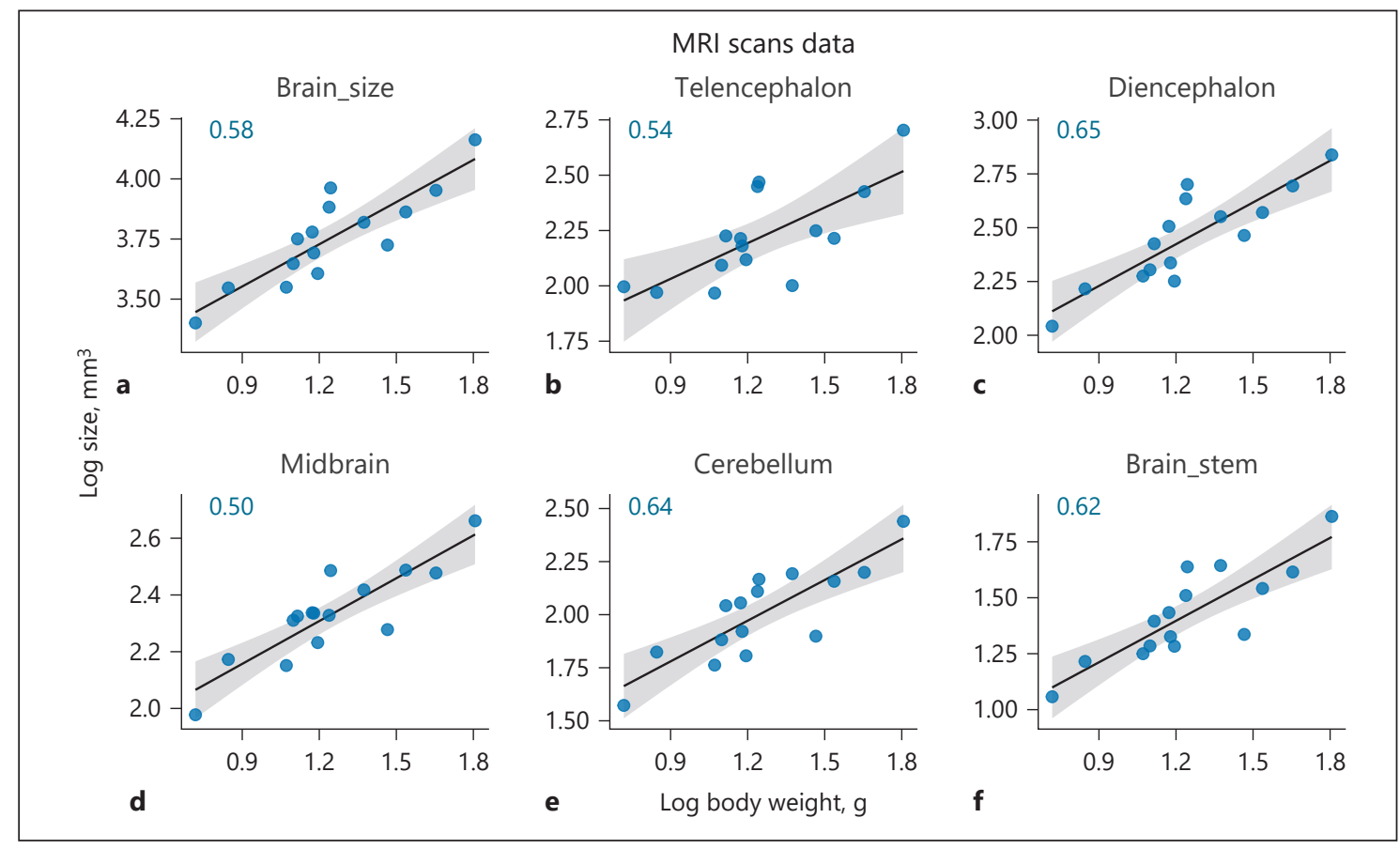

Fig. 3. Regression relationship between log-transformed total brain size (a) and log-transformed major brain part sizes (b-f) and log-transformed body weight $(\mathrm{g})$ in cleaner fish. Total brain and brain parts volume $\left(\mathrm{mm}^{3}\right)$ were estimated from MRI scans (data from Triki et al. [2019a]). The shaded area around the regression lines refers to the $95 \%$ CI. Slope estimates are also depicted in the different Figure panels.

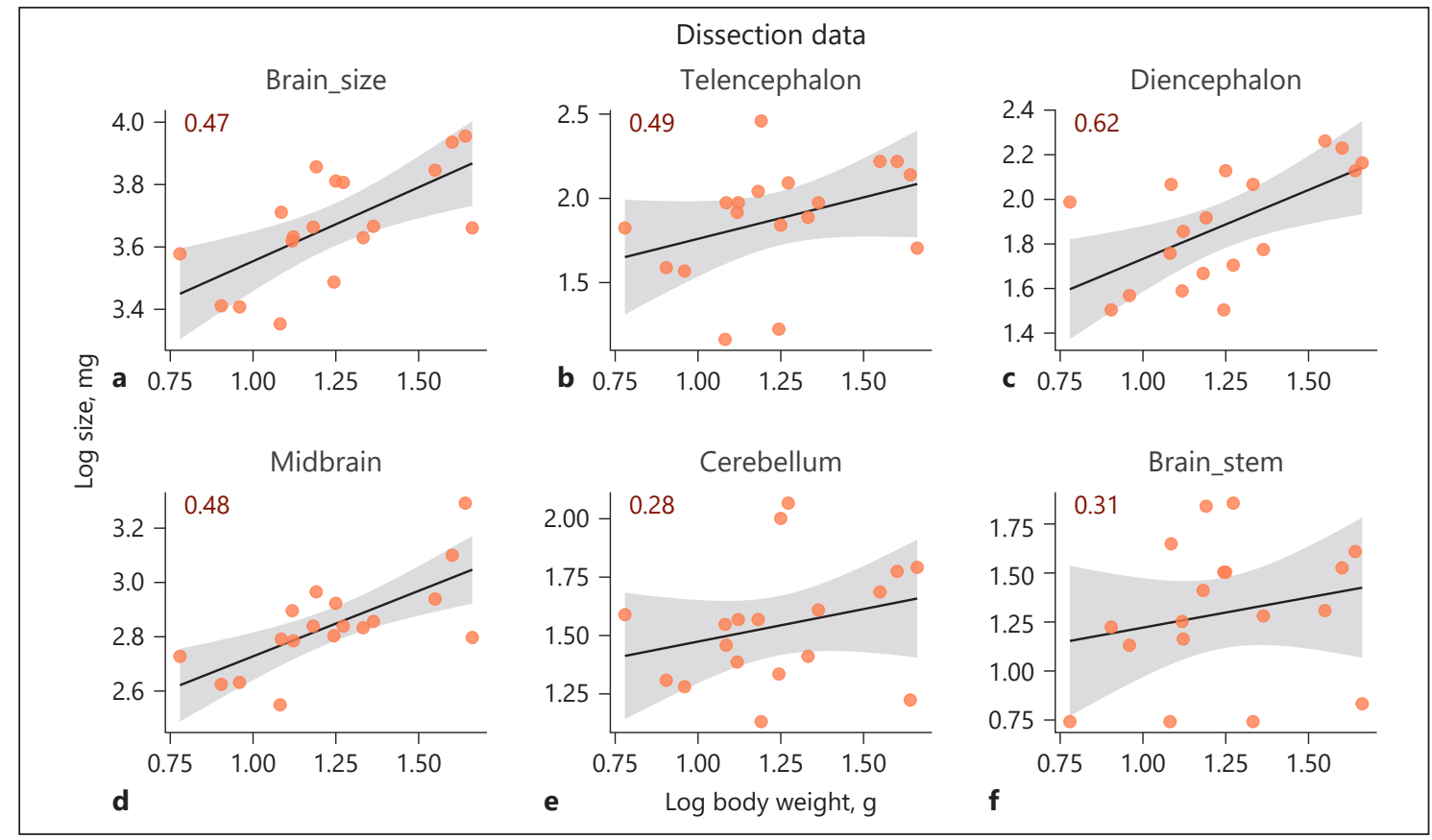

Fig. 4. Regression relationship between log-transformed total brain size (a) and log-transformed major brain part size (b-f) and log-transformed body weight ( $\mathrm{g}$ ) in cleaner fish. Total brain and brain parts mass (mg) were estimated from manual dissection and weighing. The shaded area around the regression lines refers to the $95 \%$ CI. Slope estimates are also depicted in the different Figure panels. 
Fig. 5. Relationship between cognitive performance and log-transformed body size (g) in cleaner fish (data from Aellen et al. [2021, preprint]). Regression slope and 95\% CI (grey shading) of log-transformed body weight and learning abilities in a reversal learning task (a); inhibitory control abilities in a detour task (b); numerical abilities in a numerical quantity discrimination task (c); and inhibitory control abilities in a feeding against the preferences task (d). All $y$-axes represent performance as rank, where high values on the $y$-axes refer to higher performance. In all 4 panels, $p$ values $>0.05$ were estimated from GLMs.

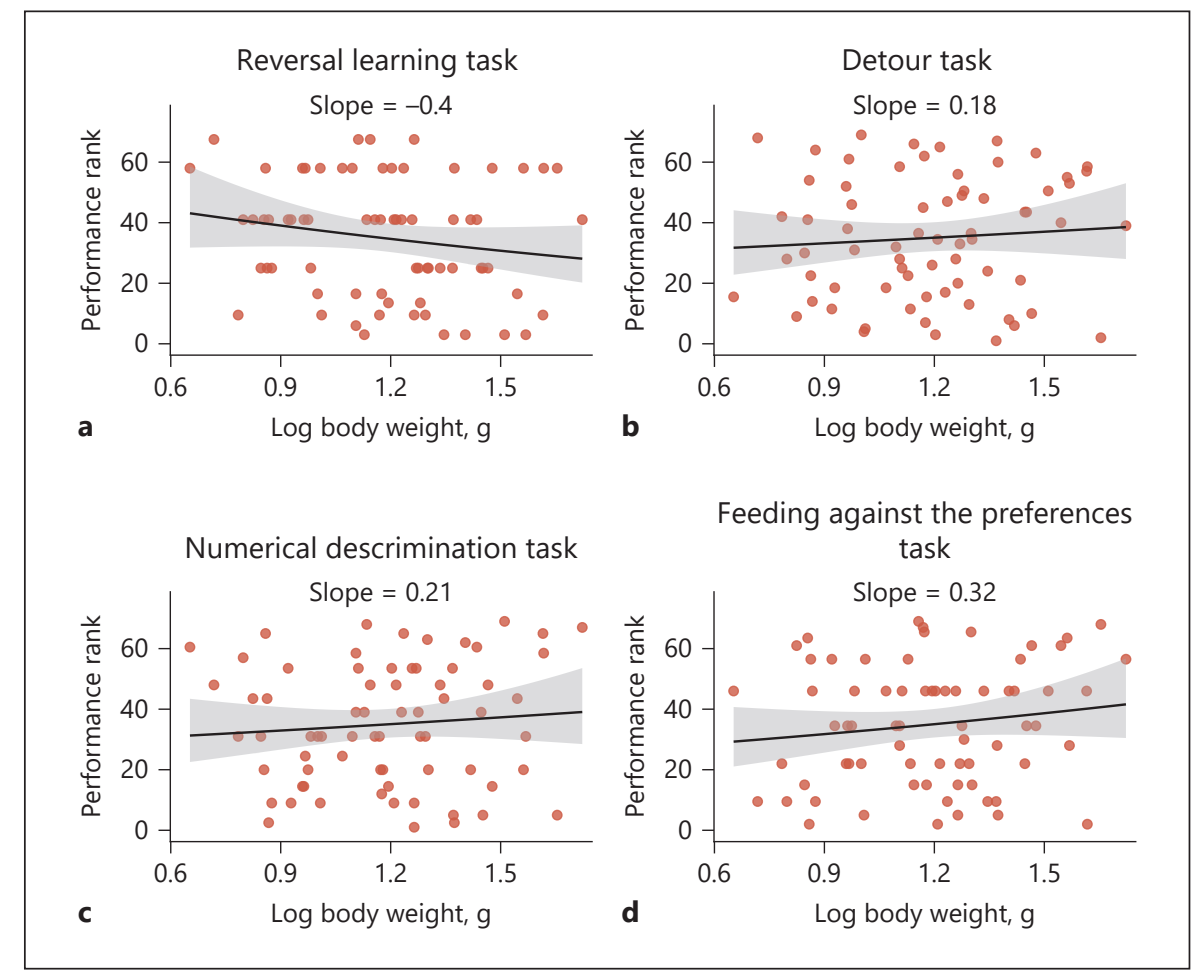

and feeding against the preferences task (GLM: $n=69, \beta$ $=0.327, t$ value $=1.181, p=0.242,95 \%$ CI $[-0.22$ to 0.87$]$; Fig. 5d).

\section{Discussion}

We first asked to what extent intraspecific slopes of the relationship between body and brain measures are higher in fishes than in mammals and to what extent variation in adult body size contributes to the differences. Because the results obtained by Tsuboi et al. [2018] might have been biased, we repeated their analysis with a restrictive dataset. This exercise closely replicated their findings and revealed no apparent effect of adult body size variation in fishes. We then asked whether intraspecific slopes provide a useful hypothesis regarding cognitive equivalence within a fish species and potentially between fish species. The key result in a case study on cleaner fish L. dimidiatus was that individual performance in 4 cognitive tasks was independent of body size. Consequently, the observed brain-body slopes (Fig. 3,4 ) of $L$. dimidiatus - which are close to the average for all fish species - most likely reflect how much brain size must be increased when the body grows to deal with somatic maintenance processes with-
Table 2. Brain-body slope estimate and brain part-body slope in the cleaner fish L. dimidiatus with $95 \% \mathrm{Cl}$

\begin{tabular}{llllll}
\hline Methods & Brain measure & $N$ & $\beta$ & Cl (lower) & Cl (upper) \\
\hline MRI, volume inTotal brain size & 15 & 0.58 & 0.38 & 0.79 \\
$\mathrm{~mm}^{3}$ & Telencephalon & 15 & 0.54 & 0.23 & 0.85 \\
& Diencephalon & 15 & 0.65 & 0.41 & 0.88 \\
& Midbrain & 15 & 0.5 & 0.33 & 0.67 \\
& Cerebellum & 15 & 0.64 & 0.38 & 0.89 \\
& Brain stem & 15 & 0.62 & 0.39 & 0.85 \\
\hline Manual & Total brain size & 18 & 0.47 & 0.19 & 0.75 \\
dissection, & Telencephalon & 18 & 0.49 & -0.16 & 1.14 \\
mass in mg & Diencephalon & 18 & 0.62 & 0.19 & 1.05 \\
& Midbrain & 18 & 0.48 & 0.23 & 0.74 \\
& Cerebellum & 18 & 0.28 & -0.24 & 0.8 \\
& Brain stem & 18 & 0.31 & -0.43 & 1.04 \\
\hline
\end{tabular}

out affecting cognitive performance. Below, we discuss how these findings can have major implications for our understanding of the evolution of cognitive performance within vertebrates.

\section{Brain-Body Slopes in Fishes}

Restricting data to species that included only wildcaught specimens and a minimal sample size of 10 indi- 
viduals, we found an average intraspecific brain-body slope of 0.46 for Actinopterygii, compared to 0.44 in Tsuboi et al. [2018]. The small negative effect of sample size on the reported slope is difficult to interpret as there is no obvious publication bias in our data. Importantly, the effect is small and, hence, does not affect the general conclusions presented above. Furthermore, the absence of an effect of adult size variation on the slope estimate indicates that there is at most a minor effect of measurement error on slope estimates. Thus, our revaluation of intraspecific brain-body slopes in fishes revealed the robustness of the slope estimates presented by Tsuboi et al. [2018] based on their much larger but less conservative dataset. This finding, therefore, corroborates that fish brain-body slopes (and, as suggested by Tsuboi et al. [2018], probably those of other ectotherms, too) are far steeper than those of birds and mammals [see also van Schaik et al., 2021].

There was an important variation of slope estimates among fish species, with minor effects of phylogenetic proximity. This suggests that targeted studies on specific fish species need to measure the slope for the species in question to generate predictions regarding the cognitive performance of individuals of this species. In our view, the average slope estimate of 0.46 is useful only for big comparative questions regarding vertebrate brains. Therefore, a major question is whether intraspecific slopes in fishes represent the slope of cognitive equivalence, as they do in mammals.

Our study also confirms the absence of a taxon-level effect in Actinopterygii reported by Tsuboi et al. [2018], with the brain-body slopes for class, order, family, genus and species being $0.50 \pm 0.01,0.51 \pm 0.03,0.49 \pm 0.02,0.50$ \pm 0.03 and $0.44 \pm 0.02$ (mean $\pm \mathrm{SE}$ ), respectively. This confirmation for fishes contrasts with the taxon-level effect in mammals found by Tsuobi et al. [2018] and recently confirmed for primates [van Schaik et al., 2021]. This finding, too, raises a major question concerning the use of EQs in fishes. We will discuss these two major questions in turn.

\section{A Case Study on Brain-Body Slopes and Cognitive Performance in Cleaner Fish}

We used existing datasets on L. dimidiatus to obtain the first preliminary estimates of the correlations between adult body size and cognitive performance and between adult body size and brain (region) size. Even though the 2 brain slope datasets available for L. dimidiatus yielded much higher values than birds and mammals, we did not find any systematic relationships be-

Cognitive Equivalence in Fishes tween an individual's body size and performance in four different cognitive tasks. The tasks we used here tested various important cognitive domains, such as flexibility in the reversal learning task, self-control in the detour task and number skills in the numerical discrimination task [Burkart et al., 2017; Aellen et al., 2021, preprint]. The fourth task - the ability to feed against preference is of high ecological relevance for cleaner fish in their interactions with client fish [Grutter and Bshary, 2003]. This task tested whether cleaner fish had prior knowledge about the consequences of eating highly preferred food (i.e., biting off clients' mucus). More precisely, with an estimated number of cleaning interactions for a single cleaner ranging between 800 and 3,000 per day [Grutter, 1995; Wismer et al., 2014; Triki et al., 2018; 2019b], all cleaner fish subjects must have learned over hundreds of thousands of interactions that eating parasites off clients has no negative effects while eating client mucus often leads to the client terminating the interaction [Bshary and Grutter, 2002]. Thus, performance in our tasks was unlikely to be affected by experience. Total brain size in the analyses could have been misleading because the tasks did not address particular challenges in perception and execution of decisions but rather learning and decision-making. However, our analyses on the five major brain region-body slopes revealed strong positive correlations in all slopes, especially with a more accurate measurement technique like MRI. Thus, our conclusions regarding the slope of cognitive equivalence do not change much, whether we take the total brain or particular regions, such as the telencephalon.

As we do not have brain data and performance data from the same fish, we could not evaluate whether individuals with a brain size above or below the slope perform above or below average. This remains an important task for future cognitive studies using cleaner fish as a study model. Until now, only one study on cleaner fish investigated performance and forebrain size in an ecologically relevant task, reporting that individuals demonstrating locally adaptive decision rules had relatively larger forebrains [Triki et al., 2020a]. These results are indicative but not conclusive with respect to our framework. However, other studies reported that cognitive abilities in guppies in a reversal learning and a detour task increase with brain size or telencephalon size [Buechel et al., 2018; Triki et al., 2021b]. Taken together, although our dataset is preliminary, the current evidence conforms with the hypothesis that the brain-body slope also represents the slope yielding cognitive equivalence in cleaner fish. Future research should conduct similar 
experiments on a variety of fish species, in particular on species that exhibit either very high or very low values of intraspecific slopes.

\section{Implications for Ectotherm-Endotherm Differences}

In combination with a recent study on brain-body slopes in primates [van Schaik et al., 2021], the results presented here have fundamental implications for predictions on the relationship between absolute brain size, brain size relative to body size and cognitive abilities across major vertebrate lineages. The primate study, which has taken extra care to reduce the effects of any measurement errors, found that intraspecific cognitive equivalence is achieved with a brain-body slope of 0.25 0.30 [van Schaik et al., 2021]. This means that if the results for cleaner fish are representative for Actinopterygii, bony fishes need, on average, an increase in (log) brain size with increasing $(\log )$ body size that is almost twice as steep to maintain cognitive equivalence than primates do. By extension, we hypothesise that ectotherm vertebrates systematically need steeper brain-body slopes than endotherm vertebrates do to achieve cognitive equivalence. This is because a strong taxon-level effect has been shown for mammals (0.25-0.3 for species according to our estimates, 0.59 for class) and birds (approximately 0.15 for species, which is not corrected for measurement error and thus too low, 0.57 for class) [Tsuboi et al., 2018], while it is absent or considerably smaller in the major ectotherm vertebrate lineages (Chondrichthyes: 0.5 for species, 0.41 for class; amphibians: 0.36 for species, 0.46 for class; nonavian reptiles: 0.41 for species, 0.56 for the class). In conclusion, our preliminary tests suggest that among both ectotherms [this study] and endotherms [van Schaik et al., 2021], cognitive performance is unrelated to body size within species, even though the mean brain-body slope in mammals is around 0.25 [van Schaik et al., 2021] and in fishes is around 0.5 . If further intraspecific tests validate this assumption, this points at fundamental differences with respect to how brain organisation translates into cognitive performance between mammals and fishes, which warrant a more detailed examination.

The use of EQ as a predictor for cognitive equivalence across species has been criticised for various reasons. Some authors have proposed that absolute brain size is a better predictor than relative brain size [Rensch, 1973; Deaner et al., 2007]. Others have pointed out that some brain areas affect cognitive performance much more strongly than others [Dunbar, 1992; Sherry et al., 1989; Lefebvre et al., 1998; Healy and Rowe, 2007]. In addition, more recent analyses show that neuron cell densities may differ between major vertebrate clades like birds and mammals and between major mammalian lineages [Herculano-Houzel, 2017], which may impose important limitations on the value of gross comparisons of brain size between more distantly related species. These problems have led to the proposal that a bottom-up approach, which focuses on comparisons between closely related species, should complement the dominating literature on large-scale comparisons [Logan et al., 2018]. Finally, van Schaik et al. [2021] criticised the EQ concept largely because of the taxon-level effects reported for endothermic vertebrates. Indeed, the more we will eventually know about the various features of brain morphology, the better we will be able to link them to variation in cognitive abilities. Our statistical testing of slope values also revealed significant variation among fish species, and a future challenge will be to search for potential ecological correlates of that variation.

For endothermic vertebrates, absolute brain size, rather than EQ, has been argued to explain best the interspecific variation in cognitive performance [Deaner et al., 2007; MacLean et al., 2012; Herculano-Houzel, 2017]. It is currently impossible to distinguish between absolute brain size and the deviation from a curve with the intraspecific mean slope as a predictor for cognitive equivalence across species. This can be partly explained by the combination of low intraspecific brain-body slopes in endotherms and the inherent uncertainty of how representative the cognitive experiments for the comparative approach are. In contrast, using a similar comparative approach on fishes (and presumably other ectotherm clades) would yield highly distinct predictions as the corrective factor of the intraspecific brain-body slopes would be relatively larger than in endotherms. The fact that absolute brain size needs to be corrected for body size in intraspecific comparisons makes us optimistic that using the mean intraspecific slope may be the best predictor we currently have for cognitive equivalence in between-species comparisons, even though large amounts of variance will remain unexplained with such an approach. Interestingly, based on the preliminary results presented in our study, currently, it appears that EQ is rather equivalent to the mean value of fishes' intraspecific slopes. Thus, EQ may work well as a gross estimate of overall cognitive performance in ectothermic vertebrates in general, as opposed to endothermic vertebrates, for which its use should be discouraged [van Schaik et al., 2021]. At least the cleaner fish data suggest that large-scale interspecific comparisons may be meaningful using $\mathrm{EQ}$, that is, without more detailed knowledge on brain (part) neuron densities. 


\section{Acknowledgements}

We thank Niclas Kolm and Tsuboi Masahito for their comments on an earlier version of the manuscript.

\section{Statement of Ethics}

There is no ethics for this present study because all data were compiled from published studies by Triki et al. [2019a; 2020a; 2020b] and Aellen et al. [2021, preprint], and from FishBase [Froese and Pauly, 2019].

\section{Conflict of Interest Statement}

The authors declare that they have no conflicts of interest.

\section{Funding Sources}

This work was supported by the Swiss National Science Foundation (grant numbers: 310030B_173334/1 to R.B.; and P2NEP3_188240 to Z.T.).

\section{Author Contributions}

C.P.S. conceived the cognitive equivalence idea. Z.T. compiled the data from the different sources, analysed the data and generated the figures. Z.T. collected the cleaner fish brain size data. M.A. collected the cognitive performance data. Z.T., C.P.S., and R.B. wrote the manuscript. All authors approved the final version of the manuscript. A preprint version of this article is available on bioRxiv [Triki et al., 2021a, preprint].

\section{Data Availability Statement}

Data and code for statistical analysis and Figures are accessible at the public repository Figshare. DOI: 10.6084/m9.figshare. 8867660 .

\section{References}

Aellen M, Burkart JM, Bshary R. No evidence for general intelligence in a fish [Internet]. bioRxiv. 2021, preprint. https://doi. org/10.1101/2021.01.08.425841.

Alroy J. Cope's rule and the dynamics of body mass evolution in North American fossil mammals. Science. 1998;280:731-4.

Binning SA, Rey O, Wismer S, Triki Z, Glauser G Soares MC, et al. Reputation management promotes strategic adjustment of service quality in cleaner wrasse. Sci Rep. 2017;7:8425.

Bohn M, Eckert J, Hanus D, Haun DB. A Longitudinal Study of Great Ape Cognition: Stability, Reliability and the Influence of Individual Characteristics [Internet]. PsyArXiv. 2021, preprint. https://doi.org/10.31234/osf.io/ pdt5w.

Bshary R, Grutter AS. Experimental evidence that partner choice is a driving force in the payoff distribution among cooperators or mutualists: the cleaner fish case. Ecol Lett. 2002;5:130-

Bshary R, Grutter AS. Image scoring and cooperation in a cleaner fish mutualism. Nature. 2006;441:975-8.

Bshary R, Wickler W, Fricke H. Fish cognition: a primate's eye view. Anim Cogn. 2002;5:1-13.

Bshary R, Würth M. Cleaner fish Labroides dimidiatus manipulate client reef fish by providing tactile stimulation. Proc Biol Sci. 2001;268:1495-501.

Buechel SD, Boussard A, Kotrschal A, van der Bijl $\mathrm{W}$, Kolm N. Brain size affects performance in a reversal-learning test. Proc Biol Sci. 2018;285:20172031.
Burkart JM, Schubiger MN, van Schaik CP. The evolution of general intelligence. Behav Brain Sci. 2017 Jan;40:e195.

Clague GE, Cheney KL, Goldizen AW, McCormick MI, Waldie PA, Grutter AS. Long-term cleaner fish presence affects growth of a coral reef fish. Biol Lett. 2011;7:863-5.

Deaner RO, Isler K, Burkart J, van Schaik C. Overall brain size, and not encephalization quotient, best predicts cognitive ability across non-human primates. Brain Behav Evol. 2007;70:115-24.

Demairé C, Triki Z, Binning SA, Glauser G, Roche DG, Bshary R. Reduced access to cleaner fish negatively impacts the physiological state of two resident reef fishes. Mar Biol. 2020;167:48.

Dunbar RI. Neocortex size as a constraint on group size in primates. J Hum Evol. 1992;22(6):469-93.

Eckert GJ. Estimates of adult and juvenile mortality for labrid fishes at One Tree Reef. Mar Biol. 1987;95:167-71.

Froese R, Pauly D. Editors. 2019. FishBase. World Wide Web electronic publication. Version $02 / 2019$. Available from: www.fishbase.org.

Froese T, Froese R. Comparisons of static brain body allometries across vertebrates must distinguish between indeterminate and determinate growth. Nat Ecol Evol. 2019 Oct;3(10):1404

Grutter AS. Relationship between cleaning rates and ectoparasite loads in coral reef fishes. Mar Ecol Prog Ser. 1995;118:51-8.

Grutter AS. Cleaner fish really do clean. Nature. 1999;398:672-3.
Grutter AS, Bshary R. Cleaner wrasse prefer client mucus: support for partner control mechanisms in cleaning interactions. Proc Biol Sci. 2003;270:S242-4

Halley AC, Deacon TW. The developmental basis of evolutionary trends in primate encephalization. In: Evolution of Nervous Systems (2nd ed). 2017 , vol. 3. pp. 149-162. https://doi org/10.1016/B978-0-12-804042-3.00135-4.

Healy SD, Rowe C. A critique of comparative studies of brain size. Proc Biol Sci. 2007;274:453-64.

Herculano-Houzel S. Numbers of neurons as biological correlates of cognitive capabilityCurr Opin. Behav Sci (Basel). 2017;16:1-7.

Huntingford FA. Implications of domestication and rearing conditions for the behaviour of cultivated fishes. J Fish Biol. 2004;65:122-42.

Irwing P, Lynn R. Sex differences in means and variability on the progressive matrices in university students: a meta-analysis. Br J Psychol. 2005 Nov;96(Pt 4):505-24

Jerison $\mathrm{H}$. Evolution of the Brain and Intelligence. New York: Academic Press; 1973.

Jones KE, Purvis A. An optimum body size for mammals? Comparative evidence from bats. Funct Ecol. 1997;11:751-6.

Kohda M, Takashi H, Takeyama T, Awata S, Tanaka H, Asai J, et al. Cleaner wrasse pass the mark test, what are the implications for consciousness and self-awareness testing in animals? PLoS Biol. 2019 Feb;17(2):e3000021.

Krebs JR, Sherry DF, Healy SD, Perry VH, Vaccarino AL. Hippocampal specialization of food-storing birds. Proc Natl Acad Sci. 1989;86:1388-92. 
Lefebvre L, Gaxiola A, Dawson S, Timmermans S, Rosza L, Kabai P. Feeding Innovations and Forebrain Size in Australasian Birds. Behaviour. 1998;135:1077-97.

Logan CJ, Avin S, Boogert N, Buskell A, Cross FR, Currie A, et al. Beyond Brain Size: Uncovering the Neural Correlates of Behavioral and Cognitive Specialization. Comp Cogn Behav Rev. [cited 2018 Dec 20], vol. 13. pp. 55-90. Available from: http://comparative-cognition-and-behavior-reviews.org/2018/vol13 logan_et-al/.

Losey GS. Fish cleaning symbiosis: proximate causes of host behaviour. Anim Behav. 1979;27:669-85.

MacLean EL, Matthews LJ, Hare BA, Nunn CL, Anderson RC, Aureli F, et al. How does cognition evolve? Phylogenetic comparative psychology. Anim Cogn. 2012;15:223-38.

Martin RD, Harvey PH. Brain Size Allometry Ontogeny and Phylogeny [Internet]. In: Jungers WL, editor. Size and Scaling in Primate Biology. Boston (MA): Springer US; 1985. pp. 147-73.

Pagel MD, Harvey PH. The taxon-level problem in the evolution of mammalian brain size. Facts Am Nat. 1988;132:344-59.

Pilbeam D, Gould SJ. Size and scaling in human evolution. Science. 1974;186:892-901.

Poirier M-A, Kozlovsky DY, Morand-Ferron J, Careau V. How general is cognitive ability in non-human animals? A meta-analytical and multi-level reanalysis approach. Proc Biol Sci. 2020;287:20201853.

Quiñones AE, Leimar O, Lotem A, Bshary R. Reinforcement Learning Theory Reveals the Cognitive Requirements for Solving the Cleaner Fish Market Task. Am Nat. 2020 Apr;195(4):664-77.

$\mathrm{R}$ Core Team. A language and environment for statistical computing [Internet]. Vienna, Austria, R Foundation for Statistical Computing; 2020. Available from: https://www.R-project. org/.
Rensch B. Gedächtnis, Begriffsbildung und Planhandlungen bei Tieren. Parey; 1973.

Ros AF, Nusbaumer D, Triki Z, Grutter AS, Bshary R. The impact of long-term reduced access to cleaner fish on health indicators of resident client fish. J Exp Biol.2020 Dec;223(Pt 24):jeb231613.

Salwiczek LH, Prétôt L, Demarta L, Proctor D, Essler J, Pinto AI, et al. Adult cleaner wrasse outperform capuchin monkeys, chimpanzees and orang-utans in a complex foraging task derived from cleaner - client reef fish cooperation. PLoS One. 2012;7:e49068.

Sherry DF, Vaccarino AL, Buckenham K, Herz RS. The hippocampal complex of food-storing birds. Brain Behav Evol. 1989;34:308-17.

Shettleworth SJ. Cognition, evolution, and behavior. 2nd ed. Oxford, New York: Oxford University Press; 2010.

Striedter GF. Principles of brain evolution. USA: Sinauer Associates; 2005.

Triki Z, Aellen M, Schaik CV, Bshary R. Relative brain size and cognitive equivalence in fishes. Brain Behav Evol. 2021, online ahead of print.

Triki Z, Bshary R. Cleaner fish Labroides dimidiatus discriminate numbers but fail a mental number line test. Anim Cogn. 2018;21:99107.

Triki Z, Bshary R. Long-term memory retention in a wild fish species Labroides dimidiatus eleven months after an aversive event. Ethology. 2019. https://doi.org/10.1111/eth.12978.

Triki Z, Emery Y, Teles MC, Oliveira RF, Bshary R. Brain morphology predicts social intelligence in wild cleaner fish. Nat Commun. 2020a;11:6423.

Triki Z, Emery Y, Teles MC, Oliveira RF, Bshary R. Data from: Brain morphology predicts social intelligence in wild cleaner fish. Figshare. 2020b. https://doi.org/10.6084/m9.figshare.7415576.

Triki Z, Fong S, Amcoff M, Kolm N. Artificial mosaic brain evolution of relative telencephalon size improves inhibitory control abilities in the guppy (Poecilia reticulata). Evolution. 2021. https://doi.org/10.1111/evo.14405.
Triki Z, Levorato E, McNeely W, Marshall J, Bshary R. Population densities predict forebrain size variation in the cleaner fish Labroides dimidiatus. Proc Biol Sci. 2019a;286:20192108.

Triki Z, Wismer S, Levorato E, Bshary R. A decrease in the abundance and strategic sophistication of cleaner fish after environmental perturbations. Glob Change Biol. 2018;24:481-9.

Triki Z, Wismer S, Rey O, Ann Binning S, Levorato E, Bshary R. Biological market effects predict cleaner fish strategic sophistication. Behav Ecol. 2019b;30:1548-57.

Tsuboi M, van der Bijl W, Kopperud BT, Erritzøe J, Voje KL, Kotrschal A, et al. Breakdown of brain-body allometry and the encephalization of birds and mammals. Nat Ecol Evol. 2018 Sep;2(9):1492-500.

van Schaik CP, Triki Z, Bshary R, Heldstab SA. A Farewell to the Encephalization Quotient: A New Brain Size Measure for Comparative Primate Cognition. Brain Behav Evol. 2021;96:112.

Victor BC. Duration of the planktonic larval stage of one hundred species of Pacific and Atlantic wrasses (family Labridae). Mar Biol, 1986;90(3):317-26.

Waldie PA, Blomberg SP, Cheney KL, Goldizen AW, Grutter AS. Long-term effects of the cleaner fish Labroides dimidiatus on coral reef fish communities. PLos One. 2011;6:e21201.

Wismer S, Grutter A, Bshary R. Generalized rule application in bluestreak cleaner wrasse (Labroides dimidiatus): using predator species as social tools to reduce punishment. Anim Cogn. 2016;19:769-78.

Wismer S, Pinto AI, Vail AL, Grutter AS, Bshary R. Variation in Cleaner Wrasse Cooperation and Cognition: Influence of the Developmental Environment? Ethology. 2014;120(6):51931. 